\title{
Review
}

\section{Imaging Central Nervous System Demyelination and Remyelination by Positron-Emission Tomography}

\author{
Benedetta Bodini ${ }^{\mathrm{a}, \mathrm{b}}$ and Bruno Stankoff ${ }^{\mathrm{a}, \mathrm{b}, *}$ \\ ${ }^{a}$ Sorbonne Université, UPMC Paris 06, Institut du Cerveau et de la Moelle épiniére, ICM, \\ Hôpital de la Pitié Salpêtriére, Inserm UMR S 1127, CNRS UMR 7225, Paris, France \\ ${ }^{\mathrm{b}}$ APHP, Assistance Publique des Hôpitaux de Paris, Hôpitaux Saint Antoine and Pitié-Salpêtriére, \\ Paris, France
}

\begin{abstract}
Positron Emission Tomography (PET), an imaging technique based on the injection of radiotracers directed against specific biological targets within brain tissues, is a specific and sensitive technique which offers the unique opportunity to quantify myelin dynamics in the central nervous system. Several stilbene and benzothiazole derivatives have been repurposed to image myelin by PET. In demyelinating and dysmyelinating models, selected radiotracers were shown to reliably quantify demyelination and remyelination, allowing a translational approach in humans. A pilot study in subjects with active relapsing MS using PET and the most available benzothiazole derivative, $\left[{ }^{11} \mathrm{C}\right] \mathrm{PIB}$, supported the hypothesis that this technique is able to quantify myelin content in multiple sclerosis (MS) lesions and to capture dynamic demyelination and remyelination over time. This study highlighted for the first time in vivo the prognostic value of individual profiles of remyelination on the disease course. In future, the clinical application of myelin PET will be pushed forward thanks to the availability of novel fluorinated tracers for myelin, together with the setting up of non invasive quantification procedures and the use of powerful PET-MR systems. This will enable to address in vivo critical unanswered questions about the pathogenesis of remyelination, and to measure the efficacy of emerging promyelinating drugs in early-phase therapeutic trials.
\end{abstract}

Keywords: Multiple sclerosis, positron emission tomography, remyelination, stlbene and benzothiazole

Conventional MRI sequences, while very sensitive for the detection of white matter lesions in demyelinating diseases, are not specific for the measure of each of the pathological processes underlying tissue damage, as detected changes may equally reflect inflammation, oedema, extra-cellular matrix changes, myelin pathology and neuro-axonal degeneration. Therefore, many advanced MRI tools have been developed over the years with the objective to quantify more specifically the myelin compartment in the CNS. However, advanced MRI techniques,

\footnotetext{
*Correspondence to: Bruno Stankoff, MD, PhD., Institut du Cerveau et de la moelle épiniére, ICM, UPMC Univ Paris 06, UMR S 1127, and CNRS UMR 7225, Hopital Pitié-Salpêtriére, F-75013, Paris, France. E-mail: bruno.Stankoff@aphp.fr.
}

while very sensitive to microstructural changes, do not display the pathological specificity required to quantify myelin loss and repair, as they reflect changes in the physical characteristics of brain tissues, rather than specific pathological processes. Positron emission tomography (PET) is a nuclear medicine imaging technology that measures the distribution of specific ligands labeled with positron emitters in vivo: as ligands are specific for targets of interest, this imaging approach provides the highest possible specificity at the cellular and/or tissular level to investigate neurological diseases. A second key advantage of PET is that it allows an absolute quantification of tracer binding that directly reflects the concentration of the biological target 
in the tissue of interest, with excellent sensitivity to changes. Therefore, PET opens the unique perspective to directly and specifically quantify myelin dynamics in demyelinating and dysmyelinating diseases in-vivo.

\section{IDENTIFYING THE CANDIDATE PET RADIOTRACERS FOR MYELIN IMAGING}

To date, the search of promising radiotracers allowing to measure myelin dynamics has been mainly focused on the development of small radiolabelled compounds that could directly bind to myelin targets, allowing the quantification of changes in myelin content in selected regions of interest within the white matter. The first candidate described was a stilbene derivative, the 1,4-bis(p-aminostyryl)-2methoxy benzene), also named BMB, that was shown to selectively bind to myelin ex-vivo and in-vivo [1]. This compound allowed the detection of demyelinating lesions in a rodent experimental autoimmune encephalitis model of demyelination, as well as the quantification of myelin loss in two dysmyelinating mutants, the shiverer and the quacking mice. On MS brain samples, the levels of BMB staining could differentiate remyelination in shadow plaques from either demyelinated lesions or normal-appearing white matter, suggesting that this biomarker was suitable to quantify myelin loss and repair in this disease. Finally, BMB was shown to be able to cross the blood brain barrier and, once radiolabeled with carbon-11, to allow the first PET imaging of CNS myelin in non-human primates.

Following this first evidence, several compounds belonging to the same chemical class as $\mathrm{BMB}$, such as BDB [2, 3], GE3111, GE3082 [4-6] and CIC [7], were shown to have similar affinity properties for myelin. In particular, GE3111 and GE3082 were investigated for the visualization of nerves in real time during surgery [4-6], while CIC was considered as another potential candidate for in-vivo CNS myelin imaging with PET [7]. Over recent years, there has been a growing interest for another stilbene derivative, the C-11-labeled N-methyl-4,4'diaminostilbene ([(11)C]MeDAS) [8], which has been proposed to have improved characteristics compared to previously described myelin tracers. In particular, this compound has been shown to present optimal biodistribution and pharmacokinetic properties and to selectively label myelinated regions with high specificity.
One of the key open issues was the identification of the molecular target responsible for the specific binding of these compounds to myelin. This was not exclusively linked to a non-specific hydrophobic binding to the lipids contained in myelin, as experimental ex-vivo evidence showed that the binding of these tracers to the myelin structure remained preserved after lipids removal [1]. Interestingly, these tracers are all derivatives of Congo-red, a dye originally used to stain deposits of beta-amyloid in Alzheimer's disease through the binding to amyloidlike proteins characterized by five adjacent beta-sheet structures [9-11]. Their additional selective binding to myelin was therefore explained with myelin proteins and amyloid plaques possibly sharing common structures, such as multiple beta-sheets, which could be targeted by these compounds [1, 12]. Recent data have strongly supported this hypothesis, providing the quantitative evidence that myelin basic protein, a major structural myelin protein which is characterised by a multiple beta-sheet structure, is one molecular binding partner of myelin-selective fluorescent compounds such as BMB, GE3082, and GE3111 [13].

As these findings suggested the existence of a molecular target common to amyloid plaques and CNS myelin, other amyloid markers related to the benzothiazole chemical class were investigated for their potential as myelin markers [14]. The thioflavinT derivative 2-(4'-methylaminophenyl)-6hydroxybenzothiazole (Pittsburg Compound B, PIB), was the first benzothiazole derivative shown to stain myelin ex-vivo in rodent and human post-mortem brain samples. The specificity of this compound for myelin was further highlighted by the evidence of its drastic decrease in the white matter of the shiverer mouse, and the demonstration of its ability to discriminate normal appearing white matter, MS demyelinated lesions and shadow plaques in post-mortem MS brains. In non-human primates, $\left[{ }^{11} \mathrm{C}\right]$-PIB allowed to generate PET images of myelinated white matter areas, that demonstrated a greatly improved white matter/grey matter contrast compared with $\left[{ }^{11} \mathrm{C}\right]-\mathrm{BMB}$ [14]. In a longitudinal experimental study performed in rodents with focal demyelination induced by lysolecithin, while $\left[{ }^{11} \mathrm{C}\right]-$ CIC showed suboptimal kinetics for a robust imaging of myelin, both the stilbene $\left[{ }^{11} \mathrm{C}\right] \mathrm{MEDAS}$ and the benzothiazole $\left[{ }^{11} \mathrm{C}\right]-\mathrm{PIB}$ were shown to be sensitive enough for capturing myelin loss and repair $[15,16]$.

More recently, other families of compounds, either related to coumarine [17] or to the sphingosine-1- 
phosphate receptor modulator fingolimod [18, 19], have been developed for myelin imaging. It is noteworthy that further data are still required to validate the specificity of these compounds toward the myelin targets, as well as their signal-to-noise ratio on PET imaging. In particular, the specificity of these compounds should be measured not only in the healthy CNS, but also in brain tissues affected by inflammatory conditions, as it is possible that some targets are expressed both by myelin and inflammatory cells. For example, this could be the case with a S1P receptor modulator such as fingolimod, that not only acts on the S1P5 site, but also on other receptor subtypes such as the S1P1, which is strongly expressed by inflammatory cells. Therefore, in order to specifically target the S1P receptors for myelin imaging, the use of a selective S1P5 compound would be essential, as this receptor has been shown to be mostly expressed on myelin and oligodendrocytes [20].

\section{THE TRANSLATION OF MYELIN PET IMAGING TO HUMAN STUDIES}

Several candidate myelin tracers potentially suitable for in-vivo PET imaging have emerged during the last decade. The essential characteristics that are necessary for the application of one of them in a pilot study in humans, include the evidence of non-toxicity, a favourable pharmacokinetic with good CNS entrance and clearance, and an acceptable signal-to-noise ratio. The availability of $\left[{ }^{11} \mathrm{C}\right]-\mathrm{PIB}$, together with its previous use in human PET studies and proven safety [21] made it a natural candidate for the first proof of concept clinical study aimed at imaging myelin changes in humans [14]. Initially, dynamic $\left[{ }^{11} \mathrm{C}\right]$-PIB PET examinations performed in patients with MS were analysed using the standardized uptake values (SUV) as a semi-quantitative measure of the tracer uptake. The SUV analysis showed that $\left[{ }^{11} \mathrm{C}\right]-\mathrm{PIB}$ PET allowed to visualize demyelinated MS lesions, with a less pronounced reduction of the tracer uptake in gadolinium enhancing lesions than in inactive lesions. A major prerequisite for a larger clinical use of this tracer was the implementation of a full quantitative model for a non invasive accurate quantification of the tracer bound fraction to white matter. This was achieved by the development and application of a voxel-wise quantification procedure based on the extraction of an input function from a reference region using a supervised clustering algorithm, followed by the application of the Logan reference tissue model [22]. This method, first developed for the TSPO [11C]PK11195 tracer [23] and subsequently applied to [11C]PIB in Alzheimer disease [24], returned parametric maps of [11C]PIB binding across the brain [25]. Interestingly, the quantitative measures of white matter brain [11C]PIB binding obtained from a group of healthy controls scanned with a High Resolution Research Tomograph (HRRT), when combined with data from the Allen brain atlas, showed a strong correlation with the mRNA expression of most of the major proteins composing the structure of human myelin, but no correlation with the mRNA expression of proteins not related to myelin. Moreover, when this non-invasive quantification method was compared to SUV in a testretest study, it showed a higher reproducibility with an ICC $>0.95$ and a test/retest absolute mean of relative differences equal to $2 \% \pm 1 \%$ [25].

We further performed a longitudinal study of myelin dynamics in patients with MS using [11C]PIB HRRT PET combined to MRI [26]. The 20 patients included were all having a relapsing-remitting form of the disease together with at least one gadoliniumenhancing lesion with a diameter above $6 \mathrm{~mm}$ on the baseline MRI, to ensure capturing an extensive degree of active demyelination and remyelination in lesions over the follow-up. The cross-sectional analysis of patients with MS compared to healthy controls revealed a progressive reduction of [11C]PIB binding from the normal-appearing white matter to the center of MS lesions, which mirrored the post-mortem evidence of a gradient in myelin density from normalappearing tissues to the lesion core [27-29]. By contrast we found no decrease in [11C]PIB binding in the normal appearing white matter (NAWM) of patients compared to the white matter of controls, suggesting that the subtle changes described on advanced MRI sequences in the NAWM [30] are not linked to a real demyelinating component. Over the follow-up period that lasted between 2 and 4 months, negative and positive changes in [11C]PIB binding were observed in lesions (Fig. 1). We calculated and extracted from PET data several dynamic indices of myelin content change, reflecting either active demyelination, active remyelination, or the balance between the two processes. One of the key findings of this study was that [11C]PIB PET identified a high between-patient variability for the index of dynamic remyelination. This result, which had not been captured in previous MRI studies, is in line with the notion of a patient-specific "remyelination 


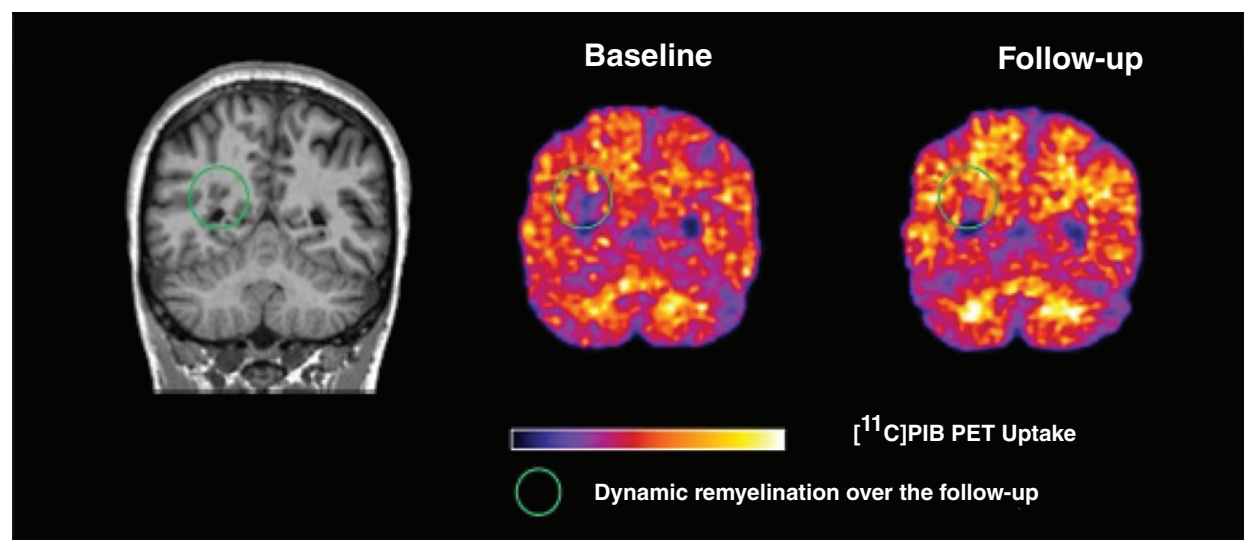

Fig. 1. Imaging myelin dynamics by PET. Left, coronal view of the MPRAGE sequence acquired on a single representative patient with relapsing-remitting MS; Centre, the corresponding [ $\left[{ }^{11} \mathrm{C}\right] \mathrm{PIB}$ PET at baseline; Right, the second PET time-point acquired 3 months later. Green circles indicate the evolution of single lesions visible as hypointense signals on MPRAGE scans, with a reduction in [11C]PIB uptake at baseline reflecting myelin loss, and a subsequent partial increase of the [11C]PIB signal at the second time-point, indicating possible dynamic remyelination.

profile," previously described in neuropathological studies [31], which could determine the extent of myelin regeneration of each individual patient in response to a demyelinating insult. A second key finding of this study was the strong association between dynamic remyelination and clinical disability scores. This evidence supports the hypothesis that an efficient remyelination process, taking place in an appropriate time window after a demyelinating insult, may be a critical factor in determining a favourable prognosis in patients with MS, at least during the relapsing phase of the disease [26]. Interestingly, recent evidence combining myelin PET and multimodal MRI, showed that patients with a higher remyelination potential were characterized by less axonal damage in white matter tracts as reflected by the measure of fractional anisotropy on diffusion weighted images, and by less grey matter damage as reflected by the measure of thalamic volume (Bodini et al., in preparation). The use of myelin PET also opens the perspective of the stratification of patients according to the individual efficiency of endogenous remyelination in a clinical setting, which may represent the first step towards personalized regenerative therapies.

\section{THE FUTURE OF MYELIN PET IMAGING IN CLINICAL STUDIES}

The exciting opportunity offered by PET encourages to push forward the research of optimized PET compounds for myelin, which would allow a larger dissemination of this technique in multicentre clinical studies of remyelination. This would imply the characterization and availability of fluorinated compounds for myelin imaging, the determination of a standardized procedure for PET image acquisition in a multicenter setting, as well as the definition of validated non-invasive methodologies aimed at quantifying the PET signal. A new generation stilbene derivative named MEDAS with improved characteristics has been recently described [32], and its fluorinated version might represent one promising candidate. Beside the development of new tracers, several stilbene and benzothiazole derivatives have already been generated in their fluorinated form and applied to clinical studies, and might be repurposed for myelin imaging as they all showed an optimal white matter uptake in control subjects [33]. Further supporting this possibility, a recent cross sectional study using PET and $\left[{ }^{18} \mathrm{~F}\right]$ Florbetaben has been performed in patients with MS, which showed that this tracer might be able to quantify myelin loss in the white matter [34]. Another major methodological step forward will be to take advantage of the synergistic combination of PET- and MRI-derived metrics, that should allow to minimize partial volume effect inherent to PET imaging, and to obtain an accurate between-modality coregistration. In this respect, novel PET-MRI systems will find an ideal application in MS, as the minimization of all methodological biases is essential to enable an accurate investigation of the myelin dynamics characterizing 
the dissemination of small lesions which is typical of this disease. The combination of PET and multimodal MRI will also enable to use PET metrics as a gold standard for myelin, and to subsequently identify and validate the best combination of MRI metrics that would correlate with the PET signal: such a combination of MRI sequences might therefore replace PET to monitor myelin dynamics in large studies, or could be associated with PET to obtain a multimodal specific and sensitive longitudinal imaging of myelin with improved spatial resolution and reduced partial volume effect. These technical improvements will finally result in the design of new early phase therapeutical trials aimed at evaluating the large number of emerging promyelinating drugs. From the quantitative data obtained in our pilot study, we suggest a design for phase II clinical trials in active RRMS that includes a run-in period lasting 4 months, to define the endogenous remyelination profiles of patients, followed by a 4 months phase with parallel arms, to evaluate the candidate molecule compared to placebo. Beyond therapeutical trials, quantitative myelin imaging technologies will help to address key unanswered questions linked to in-vivo remyelination in MS: is myelin repair a protective process preventing neuro-axonal degeneration in progressive patients? When should a promyelinating therapy be introduced during the course of the disease to maximise its effects? Is there a regional pattern of remyelination? Could the individual remyelination potential be predicted by other biomarkers?

Overall PET has several major advantages over existing imaging tools in term of specificity, sensitivity, and quantification robustness for the investigation of myelin dynamics. Ongoing optimizations of myelin PET procedures will allow a larger clinical use in the very near future, opening the perspective to disentangle the remyelination process in vivo, and to develop therapeutic trials.

\section{ACKNOWLEDGMENTS}

We thank Emilie Poirion, PhD student at the ICM, for her technical help, Mattia Veronese, King's College London, for his help to generate the figure.

We are grateful to the whole staff of the Centre d'Investigation Clinique of the ICM in Paris, and of the Service Hospitalier Frederic Joliot, CEA, Orsay. We are very grateful to ARSEP, ECTRIMS and ANR MNP2008-007125, which have supported B. Bodini with research fellowships. PET studies of remyelination have been funded by ELA (European Leukodystrophy Association, grant 2007-0481), and INSERM-DHOS (grant 2008-recherche clinique et translationnelle), and sponsored by APHP (Assistance Publique des Hopitaux de Paris). Completing this studies was possible also thanks to the support of the IHU and the following grants: Institut des neurosciences translationnelle - ANR-10-IAIHU-06.

\section{CONFLICT OF INTEREST}

The authors declare no conflict of interest.

\section{REFERENCES}

[1] Stankoff B, Wang Y, Bottlaender M, Aigrot MS, Dolle F, Wu C, et al. Imaging of CNS myelin by positron-emission tomography. Proc Natl Acad Sci U S A. 2006;103(24): 9304-9.

[2] Wu C, Tian D, Feng Y, Polak P, Wei J, Sharp A, et al. A novel fluorescent probe that is brain permeable and selectively binds to myelin. J Histochem Cytochem. 2006;54(9):9971004.

[3] Wu C, Wang C, Popescu DC, Zhu W, Somoza EA, $\mathrm{Zhu}$ J, et al. A novel PET marker for in vivo quantification of myelination. Bioorg Med Chem. 2010;18(24): 8592-9.

[4] Cotero VE, Siclovan T, Zhang R, Carter RL, Bajaj A, LaPlante NE, et al. Intraoperative fluorescence imaging of peripheral and central nerves through a myelin-selective contrast agent. Mol Imaging Biol. 2012;14(6):708-17.

[5] Gibbs-Strauss SL, Nasr KA, Fish KM, Khullar O, Ashitate Y, Siclovan TM, et al. Nerve-highlighting fluorescent contrast agents for image-guided surgery. Mol Imaging. 2011; 10(2):91-101.

[6] Gray DC, Kim EM, Cotero VE, Bajaj A, Staudinger VP, Hehir CA, et al. Dual-mode laparoscopic fluorescence image-guided surgery using a single camera. Biomed Opt Express. 2012;3(8):1880-90.

[7] Wang Y, Wu C, Caprariello AV, Somoza E, Zhu W, Wang $\mathrm{C}$, et al. In vivo quantification of myelin changes in the vertebrate nervous system. J Neurosci. 2009;29(46): 14663-9.

[8] Wu C, Wang C, Popescu DC, Zhu W, Somoza EA, Zhu J, Condie AG, Flask CA, Miller RH, Macklin W, Wang Y. A novel PET marker for in vivo quantification of myelination. Bioorg Med Chem. 2010;18(24):8592-9.

[9] Glenner GG, Eanes ED, Page DL. The relation of the properties of Congo red-stained amyloid fibrils to the -conformation. J Histochem Cytochem. 1972;20(10): 821-6.

[10] Klunk WE, Bacskai BJ, Mathis CA, Kajdasz ST, McLellan ME, Frosch MP, et al. Imaging Abeta plaques in living transgenic mice with multiphoton microscopy and methoxyX04, a systemically administered Congo red derivative. J Neuropathol Exp Neurol. 2002;61(9):797-805.

[11] Klunk WE, Pettegrew JW, Abraham DJ. Quantitative evaluation of congo red binding to amyloid-like proteins with a beta-pleated sheet conformation. J Histochem Cytochem. 1989;37(8):1273-81. 
[12] Ridsdale RA, Beniac DR, Tompkins TA, Moscarello MA, Harauz G. Three-dimensional structure of myelin basic protein. II. Molecular modeling and considerations of predicted structures in multiple sclerosis. J Biol Chem. 1997;272(7):4269-75.

[13] Bajaj A, LaPlante NE, Cotero VE, Fish KM, Bjerke RM, Siclovan $\mathrm{T}$, et al. Identification of the protein target of myelin-binding ligands by immunohistochemistry and biochemical analyses. J Histochem Cytochem. 2013;61(1): 19-30.

[14] Stankoff B, Freeman L, Aigrot MS, Chardain A, Dolle $\mathrm{F}$, Williams A, et al. Imaging central nervous system myelin by positron emission tomography in multiple sclerosis using [methyl-(1)(1)C]-2-(4'-methylaminophenyl)6-hydroxybenzothiazole. Ann Neurol. 2011;69(4): 673-80.

[15] de Paula Faria D, de Vries EF, Sijbesma JW, Dierckx RA, Buchpiguel CA, Copray S. PET imaging of demyelination and remyelination in the cuprizone mouse model for multiple sclerosis: A comparison between [11C]CIC and [11C]MeDAS. Neuroimage. 2014;87:395-402.

[16] Faria Dde P, Copray S, Sijbesma JW, Willemsen AT, Buchpiguel CA, Dierckx RA, et al. PET imaging of focal demyelination and remyelination in a rat model of multiple sclerosis: Comparison of [11C]MeDAS, [11C]CIC and [11C]PIB. Eur J Nucl Med Mol Imaging. 2014;41(5): 995-1003.

[17] Wang C, Wu C, Zhu J, Miller RH, Wang Y. Design, synthesis, and evaluation of coumarin-based molecular probes for imaging of myelination. J Med Chem. 2011;54(7): 2331-40.

[18] Briard E, Orain D, Beerli C, Billich A, Streiff M, Bigaud $\mathrm{M}$, et al. BZM055, an iodinated radiotracer candidate for PET and SPECT imaging of myelin and FTY720 brain distribution. Chem Med Chem. 2011;6(4):667-77.

[19] Briard E, Rudolph B, Desrayaud S, Krauser JA, Auberson YP. MS565: A SPECT tracer for evaluating the brain penetration of BAF312 (Siponimod). Chem Med Chem. 2015;10(6):1008-18.

[20] Jaillard C, Harrison S, Stankoff B, Aigrot MS, Calver AR, Duddy G, et al. Edg8/S1P5: An oligodendroglial receptor with dual function on process retraction and cell survival. J Neurosci. 2005;25(6):1459-69.

[21] Klunk WE, Engler H, Nordberg A, Wang Y, Blomqvist G, Holt DP, et al. Imaging brain amyloid in Alzheimer's disease with Pittsburgh Compound-B. Ann Neurol. 2004;55(3):306-19.

[22] Logan J, Fowler JS, Volkow ND, Wang GJ, Ding YS, Alexoff DL. Distribution volume ratios without blood sampling from graphical analysis of PET data. J Cereb Blood Flow Metab. 1996;16(5):834-40.
[23] Turkheimer FE, Edison P, Pavese N, Roncaroli F, Anderson AN, Hammers A, et al. Reference and target region modeling of [11C]-(R)-PK11195 brain studies. J Nucl Med. 2007;48(1):158-67.

[24] Ikoma Y, Edison P, Ramlackhansingh A, Brooks DJ, Turkheimer FE. Reference region automatic extraction in dynamic [(11)C]PIB. J Cereb Blood Flow Metab. 2013; 33(11):1725-31.

[25] Veronese M, Bodini B, Garcia-Lorenzo D, Battaglini M, Bongarzone S, Comtat C, et al. Quantification of [(11)C]PIB PET for imaging myelin in the human brain: A test-retest reproducibility study in high-resolution research tomography. J Cereb Blood Flow Metab. 2015;35(11):1771-82.

[26] Bodini B, Veronese M, Garcia-Lorenzo D, Battaglini M, Poirion E, Chardain A, et al. Dynamic imaging of individual remyelination profiles in multiple sclerosis. Ann Neurol. 2016.

[27] De Groot CJ, Ruuls SR, Theeuwes JW, Dijkstra CD, Van der Valk P. Immunocytochemical characterization of the expression of inducible and constitutive isoforms of nitric oxide synthase in demyelinating multiple sclerosis lesions. J Neuropathol Exp Neurol. 1997;56(1):10-20.

[28] Moore GR, Laule C, Mackay A, Leung E, Li DK, Zhao $\mathrm{G}$, et al. Dirty-appearing white matter in multiple sclerosis: Preliminary observations of myelin phospholipid and axonal loss. J Neurol. 2008;255(11):1802-11.

[29] Seewann A, Kooi EJ, Roosendaal SD, Barkhof F, van der Valk P, Geurts JJ. Translating pathology in multiple sclerosis: The combination of postmortem imaging, histopathology and clinical findings. Acta Neurol Scand. 2009;119(6):349-55.

[30] Miller DH, Thompson AJ, Filippi M. Magnetic resonance studies of abnormalities in the normal appearing white matter and grey matter in multiple sclerosis. J Neurol. 2003;250(12):1407-19.

[31] Patrikios P, Stadelmann C, Kutzelnigg A, Rauschka H, Schmidbauer M, Laursen $\mathrm{H}$, et al. Remyelination is extensive in a subset of multiple sclerosis patients. Brain. 2006;129(Pt 12):3165-72.

[32] Wu C, Zhu J, Baeslack J, Zaremba A, Hecker J, Kraso J, et al. Longitudinal positron emission tomography imaging for monitoring myelin repair in the spinal cord. Ann Neurol. 2013;74(5):688-98.

[33] Kepe V, Moghbel MC, Langstrom B, Zaidi H, Vinters HV, Huang SC, et al. Amyloid-beta positron emission tomography imaging probes: A critical review. J Alzheimers Dis. 2013;36(4):613-31.

[34] Matias-Guiu JA, Cabrera-Martin MN, Matias-Guiu J, Oreja-Guevara C, Riola-Parada C, Moreno-Ramos T, et al. Amyloid PET imaging in multiple sclerosis: An (18) F-florbetaben study. BMC Neurol. 2015;15:243. 